\title{
Bacteriological profile and antibiotic susceptibility patterns of lower respiratory tract infections in a tertiary care hospital, Central Kerala
}

\author{
Regha IR ${ }^{1, *}$, Sulekha B. ${ }^{2}$ \\ ${ }^{\mathbf{1}}$ Associate Professor, ${ }^{2}$ Professor \& HOD, Dept. of Microbiology, Sreenarayana Institute of Medical Sciences, Ernakulam, Kerala,
} India

*Corresponding Author:

Email: reghapks@gmail.com

\begin{abstract}
Background and Objective: Lower respiratory tract infections (LRTIs) is one of the leading human diseases causing high morbidity and mortality worldwide. The prevalent etiological agents and their antimicrobial resistance patterns differs, both geographically and over time. With emerging resistance of respiratory bacterial pathogens to commonly used antibiotics, it is imperative to study their recent trends for effective management of these cases.

Materials and Methods: A retrospective, record based study was conducted on culture and sensitivity reports of lower respiratory samples obtained in the microbiology lab during $1^{\text {st }}$ January 2015- 31st December 2017. The samples were processed by standard methods for isolation and identification followed by antimicrobial sensitivity testing using Kirby Bauer disc diffusion method.

Results: 288 (26.34\%) of total 1093 samples were positive for bacterial culture. 244 (84.7\%) were gram negative bacilli (GNB) and $44(15.3 \%)$ were gram positive cocci. The predominant pathogen isolated was K.pneumoniae (31.1\%) followed by P.aeruginosa (30.2\%). The overall susceptibility of GNB was highest towards Imipenem followed by Amikacin and Piperacillin tazobactam with resistance rates of $11.5 \%, 26.2 \%$ and $31.6 \%$ respectively. Gram positive organisms exhibited highest susceptibility towards Vancomycin and Linezolid. $15.4 \%$ of Staphylococcus aureus were Methicillin resistant (MRSA).

Conclusion: Imipenem is the most sensitive antibiotic followed by Amikacin and Piperacillin tazobactam which can be used for empirical therapy for LRTI. The antibiotic therapy should be modified as per the culture and sensitivity report. Regular determinations of the type of bacterial pathogens and updation of antibiogram must be followed in every institution to aid in better patient management by helping the clinician in the judicious use of antibiotics.
\end{abstract}

Keywords: Antibiotic resistance patterns, Lower respiratory tract infections.

\section{Introduction}

Lower respiratory tract infection (LRTI) is defined as the inflammation of the respiratory tract starting from trachea to the alveoli with subsequent multiplication of an infectious agent. ${ }^{1}$ It includes bronchitis, bronchiectasis, bronchiolitis, emphysema, lung abscess, pleural effusion and pneumonia. ${ }^{2}$ Acute lower respiratory tract (ALRI) infections are among the most common infectious diseases affecting humans worldwide causing significant morbidity and mortality for all age groups. ${ }^{1,3}$ It is responsible for $4.4 \%$ of all hospital admissions and $6 \%$ of general practitioner consultations. ${ }^{4}$ It accounts for 3 to $5 \%$ of deaths in adults .Globally, about 4.2 million ALRI deaths are estimated to occur among all age groups. ${ }^{1}$ The problem is much greater in developing countries where pneumonia is the most common cause of hospital attendance in adults. ${ }^{5}$

Each type of LRTI vary in the epidemiology, pathogenesis, clinical presentation, and outcome. ${ }^{2,5}$ The major respiratory pathogens are Gram negative bacilli (GNB) like Klebsiella pneumonia (K.pneumoniae), Escherichia coli (Esch.coli), Pseudomonas aeruginosa (P.aeruginosa), Acinetobacter species, other NonFermentative Gram-Negative Bacilli (NFGNB) and gram positive organisms like Streptococcus pneumonia (Str.pneumoniae), Staphylococcus aureus (S.aureus) etc. ${ }^{2}$
However, results from various surveillance studies show wide variations in the prevalent etiological agents and their antimicrobial resistance patterns, both geographically and over time. The dramatic rise in the antimicrobial resistance among the respiratory pathogens, presumably due to the prophylactic administration of antibacterial therapy even before the availability of the culture reports, is a matter of potential concern worldwide. Failure to de-escalate the therapy after getting the culture and sensitivity report is another important reason for the drug resistance. In this context, management of these infections has become a challenge to the physicians. $^{6}$ Hence the present study was conducted to investigate the bacterial aetiology of LRTIs in our institution and to update the clinicians on the current antibiotic susceptibility pattern of these pathogens.

\section{Materials and Methods}

A retrospective,record based study was conducted on all culture and sensitivity $(\mathrm{C} / \mathrm{S})$ reports of lower respiratory samples (sputum,endotracheal aspirate and bronchoalveolar lavage) obtained in the microbiology lab of a tertiary care hospital, Central Kerala during three consecutive years ( $1^{\text {st }}$ January 2015-31st December 2017). 
Inclusion criteria: All predominant bacterial isolates from sputum, endotracheal aspirate and bronchoalveolar lavage (BAL) during the study period

\section{Exclusion criteria:}

1. Bacterial isolates from repeat culture of previously recruited patients

2. Bacterial isolates identified as commensals or contaminants

3. Mixed bacterial growth

\section{Method}

Sputum, endotracheal aspirate and BAL were collected in a sterile wide mouth container. The quality of sputum and endotracheal tube samples were assessed based on criteria laid by American Society for Microbiology (ASM). ${ }^{6}$ According to this, a reliable specimen after gram staining would have more than 25 leucocytes and fewer than 10 epithelial cells per low power field of microscope. Samples not fulfilling these criteria were rejected for repeat specimen. The undiluted sputum samples were inoculated on the culture medium using a Nichrome wire loop. The culture media used for inoculation were blood agar, chocolate agar and Mac Conkey's agar. The inoculated plates were incubated at $37^{\circ} \mathrm{C}$ for $18-24$ hours. The predominant bacterial growth obtained from sputum samples were recorded.

Endotracheal secretions were vortexed for 1 minute, centrifuged at $3000 \mathrm{rpm}$ for 10 minutes and semiquantitative culture was performed by the calibrated loop method using a wire loop of capacity $0.001 \mathrm{~mL}$. The bacterial colonies were counted. Colony counts of $\geq 10^{5} / \mathrm{mL}$ suggest potential pathogen. ${ }^{2}$

BAL were inoculated on to blood agar, chocolate agar and MacConkey's agar and brain heart infusion broth and all were incubated at $37^{\circ} \mathrm{C}$ for $18-24$ hours. From broth it was subcultured on to blood agar and MacConkey's agar and a further 24 hours of incubation was done.

Identification of the isolates were performed by standard microbiological procedures such as study of colony morphology, Gram stain reactions and a battery of standard biochemical tests. ${ }^{7}$ Antibiotic susceptibility testing was performed by Kirby-Bauer disc diffusion method on Mueller-Hinton agar and on Blood agar for fastidious organisms. After incubation at $37^{\circ} \mathrm{C}$ for $18-24$ hours, the results were read and interpreted as per CLSI guidelines. ${ }^{8}$ The antibiotic discs used were Ampicillin $(10 \mu \mathrm{g})$, Amoxycillin-clavulanate $(20 / 10 \mu \mathrm{g})$, Piperacillin $(100 \mu \mathrm{g}), \quad$ Piperacillin-Tazobactam $\quad(100 / 10 \mu \mathrm{g})$, Gentamicin $(10 \mu \mathrm{g})$, Amikacin $(30 \mu \mathrm{g})$,Cefazolin(30 $\mu \mathrm{g})$,Ceftriaxone $(30 \mu \mathrm{g})$, Cefuroxime $(30 \mu \mathrm{g})$, Cefoxitin $(30 \mu \mathrm{g})$, Cefepime $(30 \mu \mathrm{g})$, Ceftazidime $(30 \mu \mathrm{g})$, Cotrimoxazole $(1.25 / 23.75 \mu \mathrm{g})$, Tetracycline $(30 \mu \mathrm{g})$, Ciprofloxacin(5), Imipenem(10), Penicillin(10U), Erythromycin(15 $\mu \mathrm{g})$, Clindamycin $(2 \mu \mathrm{g})$, Linezolid(30 $\mu \mathrm{g})$, and Vancomycin $(30 \mu \mathrm{g})$. Methicillin resistant Staphylococcus aureus (MRSA) were detected using Cefoxitin $30 \mu \mathrm{g}$ disc .Strains with zone size $\leq 21 \mathrm{~mm}$ were considered as MRSA. ${ }^{8}$ Penicillin sensitivity of Str. pneumoniae was detected using Oxacillin $1 \mu \mathrm{g}$ disc and a zone size $\geq 20 \mathrm{~mm}$ were considered as sensitive.

For quality control of disc diffusion tests ATCC control strains of Esch.coli ATCC 25922, S.aureus ATCC 25923 and P.aeruginosa 27853 were used.

\section{Stataistical analysis}

Descriptive statistics was used for analysis. The collected data was entered in MS-Excel and statistical analysis was done using SPSS 17 software and were expressed as percentages.

\section{Results}

Out of 1093 lower respiratory samples, 288 (26.34\%) were positive for bacterial culture. Remaining samples yielded normal pharyngeal flora/ mixed flora/no growth/fungal growth. Among the bacterial isolates 244 $(84.7 \%)$ were GNB and remaining $44(15.3 \%)$ were gram positive cocci. The predominant pathogen isolated was K.pneumoniae $(31.1 \%)$ followed by P.aeruginosa $(30.2 \%)$. Among gram positive bacteria, S.aureus (4.5\%) and Strp.pyogenes $(4.5 \%)$ were predominant organisms followed by Enterococci (4.2\%).The rate of isolation of different bacterial isolates is shown in Table 1.

The antibiotic resistance pattern of GNB is presented in Table 2. The overall resistance of GNB was lowest towards Imipenem followed by Amikacin and Piperacillin- tazobactam. Resistance to first, second and third generations of cephalosporins were significantly high. Ciprofloxacin and Cefepime exhibited comparatively good activity against GNB. Among antipseudomonal cephalosporins tested, Cefepime showed the highest rate of sensitivity followed by Ceftazidime. NFGNB demonstrated higher rate of resistance to almost all groups of antibiotics including Imipenem.

Gram positive organisms showed highest sensitivity towards Vancomycin followed by Linezolid. (Table 3). $84.6 \%$ of S.aureus were resistant to penicillin. $15.4 \%$ of S.aureus isolates were Methicillin resistant (MRSA). 100 $\%$ of Strp.pyogenes and Strp.pneumoniae were sensitive to Penicillin.

\section{Discussion}

The etiological agents of LRTIs and their susceptibility patterns vary from area to area. Hospital antibiograms are mandatory to guide empirical antimicrobial therapy and are an important component of detecting and monitoring trends in antimicrobial resistance. Reliable statistics on antibiotic resistance are mandatory to control resistant pathogens. The present study provides an insight on the prevalence and the antibiogram of respiratory pathogens in Central Kerala.

In our study, the bacterial etiology for LRTI was noticed in $26.34 \%$ of samples. The isolation rates by Mishra et al, ${ }^{9}$ Salman Khan et $\mathrm{al}^{10}$ and Ramana et $\mathrm{al}^{11}$ were $44 \%, 49.3 \%$ and $39.4 \%$ respectively. Higher 
prevalence was shown by some other authors. ${ }^{6,12,13}$ Lower bacterial isolation rate may be due to improper sample collection, delay in transportation or prior antimicrobial therapy before sample collection.

In this study Gram negative bacilli were more frequently isolated than gram positive bacteria .Many other studies also found out considerable predominance of GNB among respiratory pathogens. ${ }^{2,3,5,13-16}$ The gram negative predominace might partly be due to the unequal distribution of patients with community acquired and hospital acquired infections and also due to the spread of antibiotics resistance in hospital settings. The predominant pathogen isolated was K.pneumoniae (31.1\%). This is in concordance with Ratna S, ${ }^{5}$ Verma D et al ${ }^{17}$ Madhavi et al ${ }^{18}$ and Mokkapati A et al. ${ }^{19}$ But in some other studies the predominat pathogen was P.aeruginosa followed by K.pneumoniae., ${ }^{2,10}$ P.aeruginosa was the second most common isolate in the present study as shown by Viswanath S et al. ${ }^{20}$

Among gram positive bacteria, S.aureus (4.5\%) and Str.pyogenes $(4.5 \%)$ were most frequently isolated. S.aureus was isolated as the predominant gram positive pathogen in studies by Amutha $\mathrm{C}$ et $\mathrm{al}^{13}{ }^{13} \mathrm{Egbe}$ et $\mathrm{al}^{3}$ and Anvari MS et al ${ }^{15}$ with isolation rates of $5 \%, 15.41 \%$ and $20.8 \%$ respectively. But frequency of isolation of Str.pyogenes was relatively low in their studies. ${ }^{3,13,1}$

Against GNB, the most active antibiotics were Imipenem followed by Amikacin and Piperacillin tazobactam. K.pneumoniae and P.aeruginosa showed comparatively good susceptibility rate towards these three antibiotics. Some studies foundout good activity against them but some authors pointed out higher resistance rates (Table 4). According to our findings, Imipenem followed by Amikacin and Piperacillin tazobactam are the most suitable drugs for empirical therapy for LRTI in our settings.

$46 \%$ of GNB isolates were resistant to Ciprofloxacin. Recent studies from various parts of India demonstrated high resistance rates of K.pneumoniae towards Ciprofloxacin, but the antibiotic showed comparatively good activity against P.aeruginosa(Table 4). The GNB isolates in our hospital showed high resistance to all generations of Cephalosporins.76.4\% and $70.7 \%$ of GNB were resistant to first and second generation cephalosporins respectively. They showed 65\% resistance to third generation cephalosporins. The overall resistance to Cefepime was $42.2 \%$ which is lower when compared to other generations. $53.3 \%$ of K.pneumoniae exhibited resistance to Cefepime. Some studies showed higher resistance rate to Cefepime in the range of 65$70 \% .{ }^{6,16}$ But some contemporary studies found out good activity against this antibiotic., ${ }^{5,6,16}$ Among antipseudomonal cephalosporins, Cefepime showed the best activity against the organism as noted by some other investigators. ${ }^{2,6,13}$ Higher resistance of P.aeruginosa in a range of $57-65 \%$ against Cefepime were recorded by other authors also.,15,16 Thus, in our settings, cephalosporins and fluroquinolones were found to be ineffective for empirical therapy for LRTI .They can be tried in those isolates with proven sensitivity by susceptibility testing

Gram positive organisms showed highest sensitivity towards Vancomycin followed by Linezolid. $15.4 \%$ of S.aureus isolates were Methicillin resistant (MRSA), which is lower when compared to other studies from various states of India .In a study from Chennai it was $25 \%$, from Jharkhand it was $23.29 \%$, from Manipur it was $62.06 \%, 82.7 \%$ in iran and $55.6 \%$ in Nagpur. ${ }^{5,13-16}$ Str.pyogenes showed $23.1 \%$ resistance to Erythromycin and only $7.7 \%$ resistance to clindamycin and no resistance to Penicillin was detected. This is in concordance with a recend study by Ratna $\mathrm{S}^{5}$ Str.pneumoniae was $100 \%$ sensitive to Penicillin as demonstrated by Kombade et al. ${ }^{16}$

Table 1: Distribution of bacterial isolates from respiratory specimens

\begin{tabular}{|l|c|c|}
\hline \multicolumn{1}{|c|}{ Organism } & $\begin{array}{c}\text { Number of } \\
\text { isolates (N) }\end{array}$ & $\begin{array}{c}\text { Percentage } \\
\text { of isolation }\end{array}$ \\
\hline Klebsiella pneumoniae & 90 & 31.1 \\
\hline Pseudomonas aeruginosa & 87 & 30.2 \\
\hline Other Non -fermenting GNB & 43 & 14.93 \\
\hline Staphylococcus aureus & 13 & 4.5 \\
\hline Streptococcus pyogenes & 13 & 4.5 \\
\hline Enterococci & 12 & 4.2 \\
\hline Esch.coli & 11 & 3.8 \\
\hline Streptococcus pneumoniae & 6 & 2.1 \\
\hline Citrobacter species & 4 & 1.4 \\
\hline Proteus species & 3 & 1.1 \\
\hline Moraxella cattarhalis & 3 & 1.1 \\
\hline Burkholderia cepacia & 3 & 1.1 \\
\hline Total & 288 & \\
\hline
\end{tabular}


Table 2: Antibiotic resistance patterns of Gram negative bacteria

\begin{tabular}{|c|c|c|c|c|c|c|c|c|c|}
\hline 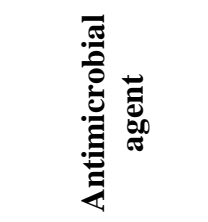 & 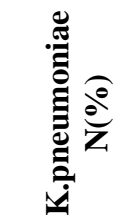 & 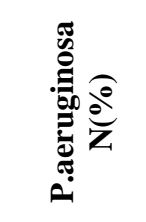 & $\begin{array}{l}\sum_{\mathrm{z}}^{0} \widehat{\mathrm{o}} \\
\mathrm{z}\end{array}$ & 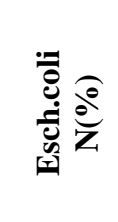 & 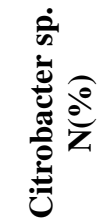 & 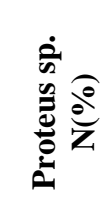 & 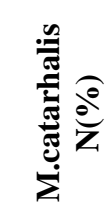 & 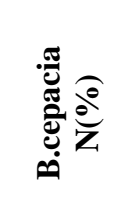 & 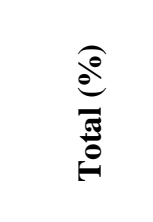 \\
\hline Ampicillin & $90(100)$ & NT & $32(74.4)$ & $8(72.7)$ & $4(100)$ & $2(66.7)$ & $0(0)$ & $0(0)$ & $136(86.6)$ \\
\hline $\begin{array}{l}\text { Amoxycillin- } \\
\text { clavulanate }\end{array}$ & $85(94.4)$ & NT & $31(72.1)$ & $7(63.6)$ & $2(50)$ & $1(33.3)$ & $0(0)$ & $0(0)$ & $126(80.3)$ \\
\hline Piperacillin & $76(84.4)$ & $27(31)$ & $27(62.8)$ & $7(63.6)$ & $4(100)$ & $0(0)$ & $0(0)$ & $0(0)$ & $141(57.8)$ \\
\hline $\begin{array}{l}\text { Piperacillin- } \\
\text { Tazobactum }\end{array}$ & $31(34.4)$ & $19(21.83)$ & $21(48.8)$ & $3(27.3)$ & $3(75)$ & $0(0)$ & $0(0)$ & $0(0)$ & 77 (31.6) \\
\hline Gentamicin & $38(42.2)$ & $43(49.4)$ & $20(46.5)$ & $4(36.4)$ & $3(75)$ & $0(0)$ & $0(0)$ & $0(0)$ & $108(44.3)$ \\
\hline Amikacin & $27(30)$ & $18(20.7)$ & $17(39.5)$ & $1(9.1)$ & $1(25)$ & $0(0)$ & $0(0)$ & $0(0)$ & $64(26.2)$ \\
\hline Cephazolin & $76(84.4)$ & NT & $34(79.1)$ & $5(45.5)$ & $4(100)$ & $1(33.3)$ & $0(0)$ & $0(0)$ & $120(76.4)$ \\
\hline Ceftriaxone & $59(65.6)$ & NT & $34(79.1)$ & $4(36.4)$ & $4(100)$ & 1(33.3) & $0(0)$ & $0(0)$ & $102(65)$ \\
\hline Cefuroxime & 71(78.9) & NT & $31(72.1)$ & $4(36.4)$ & $4(100)$ & $1(33$ & 0( & $0(0)$ & $111(70.7)$ \\
\hline Cefepime & 4 & $22(25.3)$ & $24(55.8$ & $4(36.4)$ & $4(100)$ & $1(33$ & 0( & 0 & $103(42.2)$ \\
\hline Cefoxitin & $47(52.2)$ & NT & $25(58.1)$ & $3(27.3)$ & $2(50)$ & $1(33.3)$ & 0( & $0(0)$ & $78(49.7)$ \\
\hline Ceftazidime & NT & $32(36.8)$ & NT & NT & NT & NT & 0( & NT & $32(35.6)$ \\
\hline Cefaperazone & NT & $3(37.9)$ & NT & NT & NT & NT & $0(0)$ & NT & $33(36.7)$ \\
\hline Ciprofloxacin & $46(51.1)$ & $32(36.8)$ & $25(58.1)$ & $6(54.5)$ & $2(50)$ & $0(0)$ & $0(0)$ & $1(33.3)$ & $112(46 \%)$ \\
\hline Cotrimoxazole & $55(61.1)$ & NT & $16(37.2)$ & $4(36.4)$ & $4(100)$ & $4(100)$ & $1(33.3)$ & $0(0)$ & $84(53.5)$ \\
\hline Tetracycline & $56(62.2)$ & NT & $29(67.4)$ & $7(63.6)$ & $0(0)$ & $0(0)$ & $1(33.3)$ & $0(0)$ & $93(59.2)$ \\
\hline Imipenem & $8(8.9)$ & $3(3.4)$ & $17(39.5)$ & $0(0)$ & $0(0)$ & $0(0)$ & $0(0)$ & $0(0)$ & $28(11.5)$ \\
\hline
\end{tabular}

NT: not tested

Table 3: Antibiotics resistance patterns of Gram positive cocci

\begin{tabular}{|l|c|c|c|c|c|}
\hline $\begin{array}{c}\text { Antimicrobial } \\
\text { agents }\end{array}$ & $\begin{array}{c}\text { S.aureus } \\
\mathbf{N}(\%)\end{array}$ & $\begin{array}{c}\text { Str.pyogenes } \\
\mathbf{N}(\%)\end{array}$ & $\begin{array}{c}\text { Enterococci } \\
\mathbf{N}(\%)\end{array}$ & $\begin{array}{c}\text { Str.pneumococci } \\
\mathbf{N}(\%)\end{array}$ & Total (\%) \\
\hline Penicillin & $11(84.6)$ & $0(0)$ & $8(66.7)$ & $0(0)$ & $19(43 \%)$ \\
\hline Erythromycin & $7(53.8)$ & $3(23.1)$ & NT & $2(33.3)$ & $12(37.5)$ \\
\hline Clindamycin & $2(15.4)$ & $1(7.7)$ & NT & $0(0)$ & $3(9.4)$ \\
\hline Cotrimoxazole & $0(0)$ & NT & NT & $0(0)$ & $0(0)$ \\
\hline Cefoxitin & $2(15.4)$ & NT & NT & NT & $2(15.4)$ \\
\hline Tetracycline & $2(15.4)$ & NT & NT & $0(0)$ & $2(10.5)$ \\
\hline Rifampin & $0(0)$ & NT & NT & NT & $0(0)$ \\
\hline Linezolid & $0(0)$ & $0(0)$ & $3(25)$ & $0(0)$ & $3(6.8)$ \\
\hline Vancomycin & NT & $0(0)$ & $1(8.3)$ & $0(0)$ & $1(3.2)$ \\
\hline
\end{tabular}

NT: not tested

Table 4: Resistance of K.pneumoniae and P.aeruginosa to Imipenem, Amikacin , Piperacillin tazobactam \& Ciprofloxacin in various studies

\begin{tabular}{|c|c|c|c|c|c|c|c|c|}
\hline \multirow[t]{2}{*}{ Studies } & \multicolumn{4}{|c|}{ K.pneumoniae (resistance \%) } & \multicolumn{4}{|c|}{ P.aeruginosa (resistance \%) } \\
\hline & : & 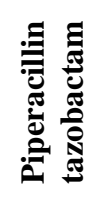 & 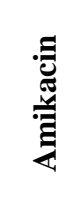 & 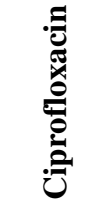 & 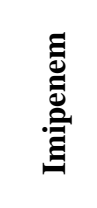 & 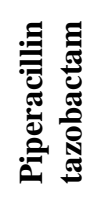 & 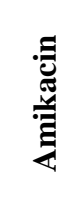 & 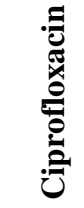 \\
\hline Presesnt study & 8.9 & 34.4 & 30 & 51.1 & 3.4 & 21.8 & 20.7 & 36.8 \\
\hline Amutha $\mathrm{C}$ et al ${ }^{13}$ & 0 & 10 & 13 & 53 & 3 & 4.4 & 30 & 42 \\
\hline Elumalai et $\mathrm{al}^{6}$ & 2.6 & 9.3 & 44.2 & 54.3 & 0 & 0 & 10.8 & 37.5 \\
\hline Ratna $S^{5}$ & 5 & 42.62 & NT & 18.03 & 7.14 & 42.85 & NT & 14.28 \\
\hline Thomas et $\mathrm{al}^{2}$ & 40 & 70 & 70 & 80 & 33.33 & 41.7 & 25 & 33.3 \\
\hline
\end{tabular}




\section{Limitations}

A distinction between community-acquired and hospital-acquired infections could not be made.

\section{Conclusion}

The study revealed GNB as major pathogens causing LRTIs. K.pneumoniae was the predominant respiratory pathogen followed by P.aeruginosa. Imipenem was the most sensitive drug, next being Amikacin and Piperacillin tazobactum and should be used for empirical therapy for LRTI. The treatment should be modified as per the culture and sensitivity report from the microbiology lab.

Antibiotic resistance among respiratory bacterial pathogens is alarming. Strict implementation of the concept of 'antibiotic stewardship' has become necessary to conserve the already available antibiotics. Hospitals should have an 'antibiotic policy' and facilities for proper monitoring of antibiotic usage along with effective infection control practices to check the issue of antibiotic resistance worldwide. Periodic analysis of types of respiratory pathogens and regular updation of their antibiograms should be done in every institutions, so that changing trends can be identified and therapy adjusted accordingly.

Funding: No funding sources.

Conflict of interest: None declared.

\section{References}

1. Pant S, Bhusal KR, Manandhar S. Microbiology of lower respiratory tract infection in workers of garment industry of Kathmandu. J Col Med Sci 2014;10(3):14-22.

2. Thomas AM, Jayaprakash C, Amma GMR. The pattern of bacterial pathogens and their antibiotic susceptibility profile from lower respiratory tract specimens in a rural tertiary care centre. J Evolution Med Dent Sci 2016;5(40):2470-76. DOI: 10.14260/jemds/2016/576

3. Egbe CA, Ndiokwre1 C, Omoregie R. Microbiology of Lower Respiratory Tract Infections in Benin City, Nigeria. Malaysian J Med Sci 2011;18(2):27-31.

4. Saxena S, Banerjee G, Garg R, Singh M, Verma1 SK, Kushwaha1 RAS. Bacterial colonization in patients with lower respiratory tract specimens: demographic profile and microbiological pattern. Int J of Med Sci Public Health 2015;1 4(11):1498-503

5. Ratna S. Bacteriological Profile and Antibiotic Susceptibility Pattern of Lower Respiratory Tract Infection in A Tertiary Hospital In North-East India. Int J Recent Sci Res 2017;8(9):20337-40. DOI: http://dx.doi.org/10.24327/ijrsr.2017.0809.0874

6. Elumalai A, Raj MA, Abarna V, Bagyalakshmi R, Reddy S. Study of Gram Negative Bacterial Isolates From Lower Respiratory Tract Infections (LRTI) and Their Antibiogram Pattern in A Tertiary Care Hospital in South India. JMSC
2015;4(11):14066-70.

DOI:https://dx.doi.org/10.18535/jmscr/v4i11.88

7. Collee, J.G., Fraser AG, Marmion BP, Simmons A. Mackey \& McCartney Practical Medical Microbiology. $14^{\text {th }}$ ed. New Delhi, India: Elsevier; 2006.

8. Performance Standards for antimicrobial susceptibility testing; Clinical and laboratory standards institute. TwentyThird Informational supplement. 2013; 33 (1):M100-S23.

9. Mishra SK, Kattel HP, Acharya J. Recent trend of bacterial aetiology of lower respiratory tract infections in a tertiary care centre of Nepal. Int J Infect Microbiol 2012;1(1):3-8.

10. Salman Khan, Singh Priti, Sachan Ankit. Bacterial Etiological agents causing LRTIs and their resistance patterns. Ir Biomed J 2015;19(4):240-46.

11. Ramana, K V. "Aetiology and Antimicrobial Susceptibility Patterns of Lower Respiratory Tract Infections (LRTI) in a Rural Tertiary Care Teaching Hospital in Karimnagar, South India." Am J Infect Dis Microbiol 2013;1(5):101-5.

12. Kaul S, Brahmadathan K, Jagannati M,Sudarsanam T, Pitchamuthu K, Abraham O. One year trends in the gram negative bacterial antibiotic susceptibility patterns in a medical intensive care unit in South India. Indian J Med Microbiol 2007;25(3):230.

13. Amutha, C., M. Suganthi, K. Radhika, K.V. Leela, J. Jayachitra and Padmanaban.Bacterial Profile of Lower Respiratory Tract Infections in Adults and their Antibiotic Susceptibility Pattern with Detection of MRSA, ESBLs and MBLs. Int J Curr Microbiol App Sci 2017;6(3):631639.doi: https://doi.org/10.20546/ijcmas.2017.603.073

14. Sharma AK, Prakash A, Kumar M .Bacterial Profile, Antibiotic Sensitivity and Resistance of Lower Respiratory Tract Infections in RIMS, Ranchi: A Tertiary Care Hospital. Int J Med Res Prof 2016;2(1):149-54.

15. Anvari MS, NaderanM, Boroumand MA Shoar S, Bakhshi R, Naderan M. Microbiologic Spectrum and Antibiotic Susceptibility Pattern among Patients with Urinary and Respiratory Tract Infection. Int J Microbiol 2014;7:682304. http://dx.doi.org/10.1155/2014/682304

16. S.P. Kombade, G.N. Agrawal. Microbiological study of lower respiratory tract infections in ICU patients. Int J Curr Microbiol App Sci 2014;3(8):749-54.

17. Verma D, Kumar P, Saxena N. Bacteriological Profile and Antibiogram Pattern in Lower Respiratory Tract Infection in Kota Region(Raj). Research \& Reviews: J Microbiol Virol 2016;6(2):13-7.

18. Madhavi S, Ramarao MV, Janardhanrao R Bacterial etiology of acute exacerbations of chronic obstructive pulmonary disease. J Microbiol Biotech Res 2012;2(3):44044.

19. Mokkapati A, Yalamanchili M. Correlation of Sputum Gram's stain and culture in lower respiratory tract infections. IOSR JDMS 2013;8(1):6-9.

20. Viswanath S, Chawla K, Gopinathan A. Multi drug resistant Gram negative bacilli in lower respiratory tract infections. Iran J Microbiol 2013;5(4):323-27.

How to cite this article: R. Regha, B. Sulekha. Bacteriological profile and antibiotic susceptibility patterns of lower respiratory tract infections in a tertiary care hospital, Central Kerala. Int J Med Microbiol Trop Dis 2018;4(4):186-90. 